\title{
Dependência térmica da germinação de sementes de Drymaria cordata (L.) Willd. ex Roem. \& Schult. (Cariophyllaceae)
}

\author{
Victor José Mendes Cardoso ${ }^{1,2}$ e Fernando Jorge Muniz Pereira ${ }^{1}$
}

Recebido em 21/01/2008. Aceito em 26/05/2008

\begin{abstract}
RESUMO - (Dependência térmica da germinação de sementes de Drymaria cordata (L.) Willd. ex Roem. \& Schult. (Cariophyllaceae)). A temperatura representa um importante fator ambiental regulador da germinação de sementes. Procurou-se avaliar a resposta de sementes de $D$. cordata à temperatura, com base no modelo de graus-dia, testando-se assim a eficiência desse modelo em descrever o comportamento germinativo da semente em diferentes regimes térmicos. Testou-se também a resposta das sementes à luz, concluindo-se que a luz branca promove a germinação. As temperaturas mínima, ótima e máxima de germinação foram, respectivamente, 17,1, 26 e $33,4{ }^{\circ} \mathrm{C}$. Considerando-se que a velocidade de germinação de $D$. cordata variou com a temperatura numa relação aproximadamente linear, o modelo de graus-dia pode ser uma ferramenta válida para se estudar a dependência da temperatura da germinação dessas sementes. Uma possível aquisição de dormência durante a incubação isotérmica pode exigir a aplicação de outros modelos que descrevam melhor o comportamento germinativo de $D$. cordata em diferentes regimes térmicos.
\end{abstract}

Palavras-chave: fotoblastismo, graus dia, planta invasora, temperatura

\begin{abstract}
Thermal-dependence of seed germination in Drymaria cordata (L.) Willd. ex Roem. \& Schult. (Cariophyllaceae)). Temperature is an important environmental factor controlling seed germination. The temperature response of $D$. cordata seeds was assessed on a thermal time basis in order to evaluate this model describing germination behavior of the seeds under different thermal regimes. Seed response to light was also tested, and the results led to the conclusion that germination was promoted by white light. Base, optima and ceiling temperatures for germination of $D$. cordata were, respectively, $17.1{ }^{\circ} \mathrm{C}, 26{ }^{\circ} \mathrm{C}$ and $33.4{ }^{\circ} \mathrm{C}$. Since germination rate was linearly related to temperature, the thermal time model can be used to assess temperature dependence of seed germination in D.cordata. Possible acquisition of seed dormancy during imbibition may not allow a good fit of cumulative germination under some temperature regimes, and other models must be used.
\end{abstract}

Key words: photoblastic reaction, temperature, thermal time, weed

\section{Introdução}

A literatura científica está repleta de trabalhos tratando do efeito de água, luz e temperatura sobre a germinação de sementes. Embora os desenhos experimentais sejam relativamente simples e de fácil execução, a análise dos resultados de ensaios de germinação nem sempre é realizada da maneira adequada, o que pode comprometer a interpretação dos dados experimentais. $\mathrm{O}$ modo mais comum de se apresentar os resultados de um ensaio de germinação é a curva de germinação acumulada (germinação $\times$ tempo). De acordo com Brown \& Meyer (1988), as curvas de germinação, com os respectivos modelos de ajuste, descrevem melhor o comportamento das sementes ao longo do tempo, do que o fazem índices isolados. Por outro lado, embora as equações derivadas desses modelos possam ser úteis para ajuste das curvas, o significado biológico de seus parâmetros muitas vezes não é claro (Welbaum et al. 1998).

As curvas de germinação indicam os tempos necessários para que as sementes individuais de uma população germinem, refletindo, portanto, a homogeneidade ou uniformidade da população de sementes quanto à distribuição dos tempos de germinação. A velocidade de germinação, por sua vez, pode ser definida como o inverso dos tempos necessários para a germinação de sementes individuais ou, na prática, grupos de sementes (Welbaum et al. 1998). Considerando-se assim o envolvimento de populações e porcentagens em experimentos de germinação, um modelo de análise que vem sendo bastante utilizado nas duas últimas décadas é aquele baseado no conceito de graus-dia (Garcia-Huidobro et al. 1982).

\footnotetext{
1 Universidade Estadual Paulista Julio de Mesquita Filho, IB, Departamento de Botânica, Campus de Rio Claro, C. Postal 199, 13506-900 Rio Claro, SP, Brasil

2 Autor para correspondência: victorjc@rc.unesp.br
} 
A resposta à temperatura $T$ pode ser caracterizada em termos das temperaturas cardeais: mínima (ou base); ótima e; máxima (ou teto). As temperaturas base (Tb) e máxima $\left(T_{M}\right)$ representam, respectivamente, os limites inferior e superior, além dos quais não ocorre germinação; enquanto a temperatura ótima ( $T o$ ) é aquela em que a germinação é maior e mais rápida (Labouriau 1983; Bradford 2002). A temperatura ótima (ou faixa ótima) divide em dois segmentos a curva de resposta das sementes à temperatura, um intervalo infra-ótimo, situado entre $T b$ e $T o$; e um intervalo supra-ótimo, localizado entre $T o$ e $T_{M}$. A aplicação da teoria de "graus-dia" à germinação leva em conta a existência de um intervalo térmico no qual a velocidade de germinação de uma determinada fração ou porcentagem $g$ de sementes apresenta uma relação linear com a temperatura. Quando isso ocorre, e quando $T b$ é praticamente a mesma para todas as frações da população, as várias isotermas podem ser convergidas para uma única curva, plotando-se a germinação contra graus-dia (thermal time ou $\theta_{g}$ ), que é o produto da multiplicação de $T-T b$ pelo tempo $\left(\mathrm{t}_{\mathrm{g}}\right)$ necessário para a germinação da porcentagem $g$ (Gummerson 1986), expresso por $\theta_{\mathrm{g}}=\left(\mathrm{T}-\mathrm{T}_{\mathrm{b}}\right) \mathrm{t}_{\mathrm{g}}$ (equação 1).

A teoria que embasa esse modelo assume que a ordem de germinação de cada semente, dentro da população, é independente da temperatura. Isso permite que as sementes sejam enquadradas em frações $g$, que funcionam como um identificador da semente. Uma fração $g=5 \%$, por exemplo, representa as sementes mais rápidas, ou seja, as primeiras a alcançarem a marca de $5 \%$ de germinação, qualquer que seja a temperatura. $\mathrm{O}$ modelo também assume que $T b$ seja relativamente constante para as diferentes frações, e que as diferenças entre as frações decorram de diferentes necessidades em termos de $\theta_{g}$. Usando-se esse método, pode-se comparar curvas de germinação obtidas em temperaturas diferentes localizadas no intervalo infra-ótimo, eliminando-se o efeito térmico sobre a distribuição dos tempos de germinação. Por outro lado, na faixa supraótima, onde a velocidade decresce com o aumento da temperatura, observa-se que, em alguns casos, $T_{M}$ varia com a temperatura, enquanto $\theta_{g}$ assume valor relativamente constante (Bradford 2002). Nesse caso, um segundo modelo apresentado por Garcia-Huidobro et al. (1982), expresso por $\theta_{g}=\left(\mathrm{T}_{\mathrm{M}}-\mathrm{T}\right) \mathrm{t}_{\mathrm{g}}$ (equação 2), pode ser usado.

O conceito de graus-dia tem-se revelado útil, permitindo boas estimativas do tempo de emergência de plântulas, a partir de informações da temperatura real do solo após a semeadura ou dispersão das sementes (Miller et al. 2001; Qiu et al. 2006). Basicamente, o modelo relaciona um dado processo de desenvolvimento com a energia térmica recebida pelo sistema. Conhecendo-se essa relação representada, no caso do processo de germinação, pelo parâmetro $\theta_{g}$, e conhecendo-se a temperatura base ou mínima $\mathrm{T}_{\mathrm{b}}$, é possível prever-se teoricamente o tempo necessário para que uma dada porcentagem das sementes de uma população germine num determinado regime térmico. A quantidade de "graus-dia" que um sistema vivo recebe no ambiente natural é representada pelo somatório dos produtos da quantidade de ${ }^{\circ} \mathrm{C}$ acima da $\mathrm{T}_{\mathrm{b}}$ pelo tempo de exposição (no caso dia). Por exemplo, se $\mathrm{T}_{\mathrm{b}}$ é igual a $10{ }^{\circ} \mathrm{C}$ e a temperatura média no dia 1 foi $11^{\circ} \mathrm{C}$ e no dia $213{ }^{\circ} \mathrm{C}$, então 4 graus-dia são acumulados ( 1 no primeiro dia e 3 no segundo).

Modelos sobre o efeito da temperatura na germinação podem ser aplicados em trabalhos visando o manejo mais adequado de plantas daninhas (Grundy et al. 2003; Battla \& Benech-Arnold 2006). Drymaria $\operatorname{cordata}($ L.) Willd. ex Roem. \& Schult. (Cariophyllaceae) é uma planta anual, herbácea, nativa da América tropical, propagando-se principalmente por sementes. Trata-se de uma invasora comum em várzeas e locais mais ou menos sombreados, infestando jardins, pastagens, hortas e terrenos baldios (Lorenzi 1982). Neste trabalho, avaliou-se a germinação de sementes de uma população de $D$. cordata em resposta à temperatura, com base no modelo de graus-dia, testando-se assim a eficiência desse modelo em descrever o comportamento germinativo da semente em relação a flutuações da temperatura. Ensaios preliminares também foram realizados para se avaliar a resposta das sementes à luz, de modo a se estabelecer um protocolo experimental mais adequado.

\section{Material e métodos}

Foram utilizadas sementes de Drymaria cordata coletadas no mês de abril/2004, em Rio Claro, SP, a partir de plantas que ocorrem espontaneamente em um terreno gramado. A coleta foi feita manualmente, sendo os frutos colocados para secar ao ar livre em bandejas, para a liberação das sementes, as quais foram então separadas por peneiramento e armazenadas em frascos de plástico protegidos da luz, à temperatura de $25^{\circ} \mathrm{C}$.

Os ensaios com temperatura isotérmica foram realizados em um equipamento para gradiente térmico, cuja construção foi baseada em modelo descrito por Labouriau \& Agudo (1987). O equipamento foi adaptado por Cardoso (inédito), com iluminação interna fornecida por lâmpadas fluorescentes que propiciam irradiância média às sementes de aproximadamente $6 \mu \mathrm{mol} \mathrm{m} \mathrm{m}^{-2} \mathrm{~s}^{-1}$. Essas foram dispostas sobre papel de filtro saturado com água destilada, dentro de recipientes de polipropileno inseridos em tubos de ensaio $(25 \times 2,5 \mathrm{~cm})$ dotados de 
tampas de inox, em metodologia baseada em Labouriau \& Agudo (1987). Cada tratamento térmico foi feito em cinco tubos contendo 100 sementes cada. Os tubos foram distribuídos de maneira aleatória dentro de cada estação térmica, formada pela sobreposição de perfilados de alumínio de seção retangular, dentro dos quais foram inseridos os tubos de ensaio com as sementes. Em sua totalidade, o bloco de gradiente térmico é constituído por 10 estações térmicas dispostas uma ao lado da outra e separadas entre si por uma placa de alumínio de 1/2". Sensores PT100 ligados a uma chave seletora digital (mod. SK 010, JK Instrumentos) foram usados no monitoramento das temperaturas nas estações térmicas. As temperaturas médias em cada estação foram, respectivamente: $13,9 \pm 0,9 ; 17 \pm 0,4 ; 20,2 \pm 0,3 ; 23,1$ $\pm 0,3 ; 26,1 \pm 0,3 ; 29 \pm 0,3 ; 31,6 \pm 0,3 ; 34,6 \pm 0,3 ; 36,8$ $\pm 0,4$; e $39,3 \pm 0,5{ }^{\circ} \mathrm{C}$.

Os ensaios com diferentes condições de luz (luz branca, escuro, luz vermelha e vermelho-extremo) foram realizados em câmara de germinação marca "Nova Técnica", modelo NT 708 AT. As sementes foram dispostas em placas de Petri de $60 \mathrm{~mm}$, sobre dois discos de papel de filtro saturados com água destilada. Foram usadas, para cada tratamento, três placas com 100 sementes cada. Para minimizar a evaporação as placas foram colocadas dentro de bandejas de plástico revestidas internamente com papel de filtro umedecido com água destilada, cobertas com uma chapa de vidro liso transparente. A condição de luz vermelha foi obtida usando-se como filtro duas folhas de papel celofane vermelho (pico de $0,012 \mathrm{~W} \mathrm{~m}^{-2} \mathrm{~nm}^{-1}$ a $650 \mathrm{~nm}$ ), enquanto que para vermelho extremo a luz foi filtrada através de duas camadas de celofane vermelho e três de azul $\left(0,013 \mathrm{~W} \mathrm{~m}^{-2} \mathrm{~nm}^{-1}\right.$ a $\left.750 \mathrm{~nm}\right)$. A condição de escuro foi obtida colocando-se as placas dentro de caixas de germinação (Gerbox) pretas. Para o tratamento sob luz branca foram usadas lâmpadas fluorescentes de $15 \mathrm{~W}$ tipo "luz do dia", com irradiância média de $29,3 \mu \mathrm{mol} \mathrm{m}{ }^{-2} \mathrm{~s}^{-1}$.

As contagens de germinação (protrusão da radícula) foram feitas diariamente. No caso dos tratamentos de escuro e vermelho extremo constante, as contagens foram feitas sob luz verde de segurança. As porcentagens finais $(\mathrm{G})$ e velocidades médias de germinação $\left(\mathrm{V}_{\mathrm{m}}\right)$ (Labouriau, 1983) foram submetidas à análise de variância e comparadas pelo teste de Tukey (Sokal \& Rohlf 1969). Os intervalos térmicos infra-ótimo, ótimo e supra-ótimo foram estimados a partir dos gráficos de $G$ e $V_{m}$ contra temperatura.

Foram elaboradas, para cada temperatura, curvas de distribuição das porcentagens médias de germinação acumulada ao longo do tempo, sendo os pontos ajustados de acordo com a função de Weibull, que é um modelo prático e adequado para se descrever curvas de germinação (Dumur et al. 1990), obedecendo a equação $\mathrm{g}=\mathrm{A}\left[1-\exp \left(-\{\mathrm{k}[\mathrm{t}-\mathrm{z}]\}^{\mathrm{c}}\right)\right]$ (equação 3 ), onde $g$ é a porcentagem de germinação, $t$ é o tempo (dias) e $A, k, z$ e $c$ são os parâmetros da equação, correspondentes respectivamente a: germinação máxima; velocidade; tempo entre o início do experimento e a germinação visível e; característica da distribuição das freqüências de germinação (Dumur et al. 1990).

Em seguida, para cada temperatura, foram determinados os tempos $\left(\mathrm{t}_{\mathrm{g}}\right)$ necessários para a germinação das frações percentuais (g) de 5, 10, 20, $30,40,50,60$ e $70 \%$, usando-se a equação 3 , rearranjada segundo a expressão $\mathrm{t}_{\mathrm{g}}=\left\{\left[\ln (\mathrm{A} /(\mathrm{A}-\mathrm{g}))^{1 / \mathrm{c}}\right] / \mathrm{k}\right\}+\mathrm{z}$ (equação 4).

A partir de $t_{\mathrm{g}}$ foram estimadas as velocidades de germinação $\left(1 / \mathrm{t}_{\mathrm{g}}\right)$ para cada uma das frações percentuais $g$ (Garcia-Huidobro et al. 1982). Em seguida, foi feito um gráfico relacionando velocidades (ordenadas) e temperaturas (abscissas), sendo os pontos ajustados por regressão linear de modo a produzir uma reta para cada fração percentual $g$. A intersecção de cada reta com o eixo $\mathrm{x}(\mathrm{y}=0)$ corresponde à $\mathrm{T}_{\mathrm{b}}$ para as temperaturas infra-ótimas, e à $T_{M}$ para as temperaturas supra-ótimas, enquanto que os valores de $\theta_{\mathrm{g}}$ são representados pelas recíprocas das inclinações das retas (Garcia-Huidobro et al. 1982). Conhecendo-se a relação entre velocidade e temperatura, as porcentagens diárias acumuladas de germinação, correspondentes a cada $t_{g}$ para as temperaturas consideradas sub-ótimas, foram transformadas em probit (recurso INV. NORM do programa Excel) e plotadas contra $\left[\left(\mathrm{T}-\mathrm{T}_{\mathrm{b}}\right) \mathrm{t}_{\mathrm{g}}\right]$ num único gráfico. Os pontos foram então ajustados de acordo com $\mathrm{o}$ modelo linear probit $(\mathrm{g})=\mathrm{a}+\left\{\log \left[\left(\mathrm{T}-\mathrm{T}_{\mathrm{b}}\right) \mathrm{t}_{\mathrm{g}}\right]\right\} / \sigma \theta$ (equação 5), onde T é a temperatura experimental, "a" é o intercepto e ó é o desvio padrão representado pelo inverso do coefíciente de inclinação da reta. Para a escolha da temperatura base $\left(T_{b}\right)$, diferentes valores de $\mathrm{T}_{\mathrm{b}}$ foram experimentados até se obter o melhor ajuste da curva (maior $\mathrm{R}^{2}$ ) (Bradford 1995). Considerando-se a aparente convergência na abscissa das retas do gráfico de velocidades contra temperatura, o mesmo procedimento foi usado para as temperaturas supraótimas para se encontrar a temperatura máxima $\left(\mathrm{T}_{\mathrm{M}}\right)$, apenas substituindo-se $\left[\left(T-T_{b}\right) t_{g}\right]$ por $\left[\left(T_{M}-T\right) t_{g}\right]$ na equação 5. Para se exibir o decurso da germinação isotérmica em escala de graus-dia, multiplicou-se o tempo (dias) pelo fator $\left(\mathrm{T}-\mathrm{T}_{\mathrm{b}}\right)$, para o intervalo infra-ótimo, ou pelo fator $\left(\mathrm{T}_{\mathrm{M}}-\mathrm{T}\right)$, para o intervalo supra-ótimo.

Os tempos esperados para a germinação nas faixas térmicas infra e supra-ótima foram estimados partir da equação 5 , isolando-se o $\mathrm{t}_{\mathrm{g}}$ conforme a expressão $\mathrm{t}_{\mathrm{g}}\left[10^{\text {(probit(g)-a). } \sigma}\right] /(\mathrm{T}-\mathrm{Tb})$, para a faixa infra-ótima, ou 
$\mathrm{t}_{\mathrm{g}}=\left[10^{\text {(probit(g)-a). } \sigma}\right] /\left(\mathrm{T}_{\mathrm{M}^{-}} \mathrm{T}\right)$, para a faixa supra-ótima. Valores observados e esperados foram comparados por teste de $\chi^{2}(\alpha=0,05)$.

\section{Resultados e discussão}

Considerando-se que a resposta de sementes à luz pode ser afetada pela temperatura (Felippe 1980; Cardoso 1990; Tobe et al. 2006), foram realizados experimentos preliminares para se verificar o efeito da luz na germinação em três temperaturas diferentes. Observouse que a luz branca (LB) favoreceu a germinabilidade a 19,25 e $32{ }^{\circ} \mathrm{C}$, apresentando valores iguais ao tratamento com luz vermelha (LV). Escuro (E) e vermelho-extremo (VE) inibiram a germinação final (Fig. 1A). Assim, é possível concluir que sementes de Drymaria cordata exibem fotoblastimo, a exemplo de inúmeras outras espécies de plantas consideradas invasoras, fazendo com que programas de controle dessas espécies levem muito em conta essa característica (Juroszek \& Gerhards
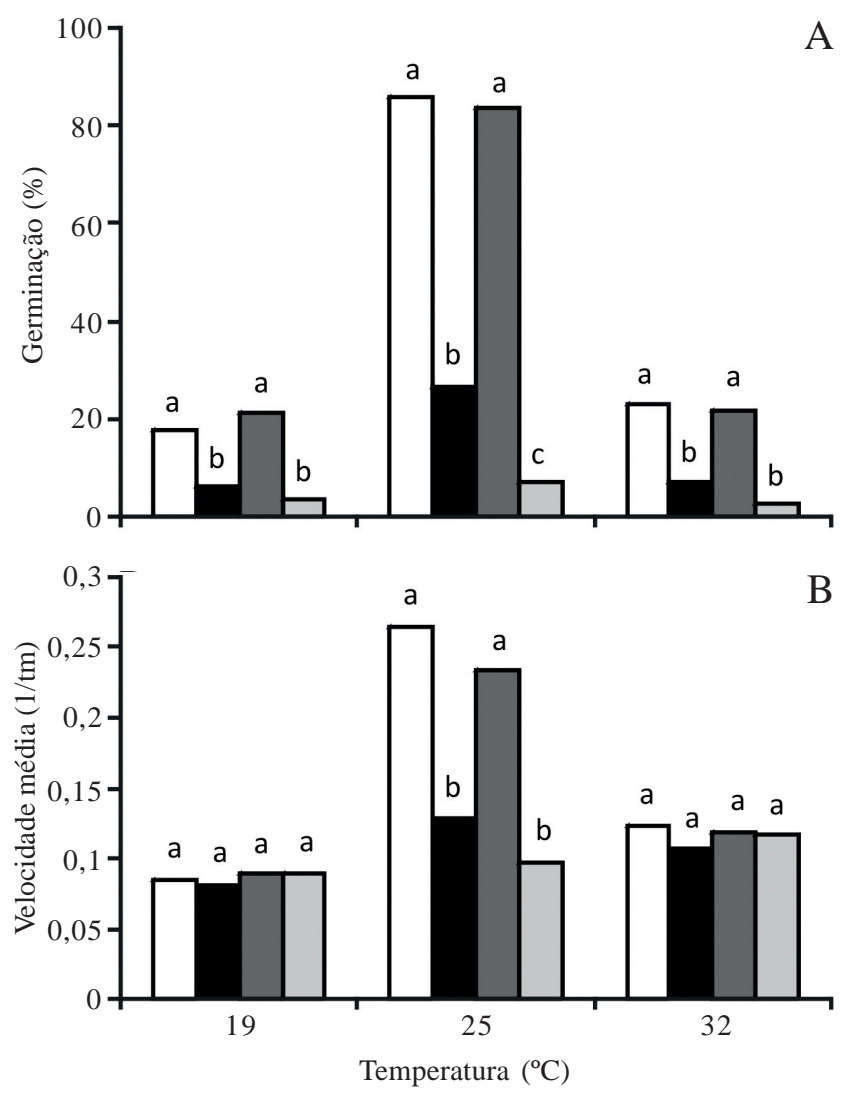

Figura 1. Efeito da luz branca (LB), escuro (E), luz vermelha (V) e vermelho extremo (VE) sobre a porcentagem (A) e velocidade média de germinação (B) de sementes de Drymaria cordata (L.) Willd. ex Roem. \& Schult. (Cariophyllaceae) às temperaturas de 19, 25 e $32{ }^{\circ} \mathrm{C}$. Velocidade média determinada como a recíproca do tempo médio $\left(\mathrm{t}_{\mathrm{m}}\right)$. Os ensaios tiveram a duração de 30 dias. Letras sobre as barras comparam as médias de luz dentro da cada regime térmico por intermédio do teste de Tukey $(\alpha=0,05 ; \mathrm{n}=3)(\square=\mathrm{LB} ; \mathbf{\square}=\mathrm{E}$; a $=\mathrm{V} ; \square=\mathrm{VE})$.
2004). Quanto à velocidade média, LB e LV promoveram a germinação apenas a $25^{\circ} \mathrm{C}$, não havendo diferença entre os tratamentos às temperaturas de 19 e $32{ }^{\circ} \mathrm{C}$ (Fig. 1B). Considerando-se esses resultados, os experimentos sobre o efeito da temperatura foram realizados no bloco termo-gradiente sob luz fluorescente branca. Constatou-se que, embora as irradiâncias médias tenham sido bem menores no termo-gradiente $\left(6 \mu \mathrm{mol} \mathrm{m} \mathrm{m}^{-2} \mathrm{~s}^{-1}\right)$ em comparação com aquelas obtidas na câmara de germinação $\left(29,3 \mu \mathrm{mol} \mathrm{m} \mathrm{m}^{-2} \mathrm{~s}^{-1}\right)$ onde o experimento para testar o efeito da luz foi realizado, aparentemente essa redução não afetou a germinação, pelo menos nas proximidades da temperatura ótima. Basta comparar as germinabilidades sob luz branca na câmara de germinação, $85,7 \%$ a $25^{\circ} \mathrm{C}$ (Fig. 1A), e no gradiente, $83,7 \%$ à temperatura de $26,1^{\circ} \mathrm{C}$ (Fig. 2).

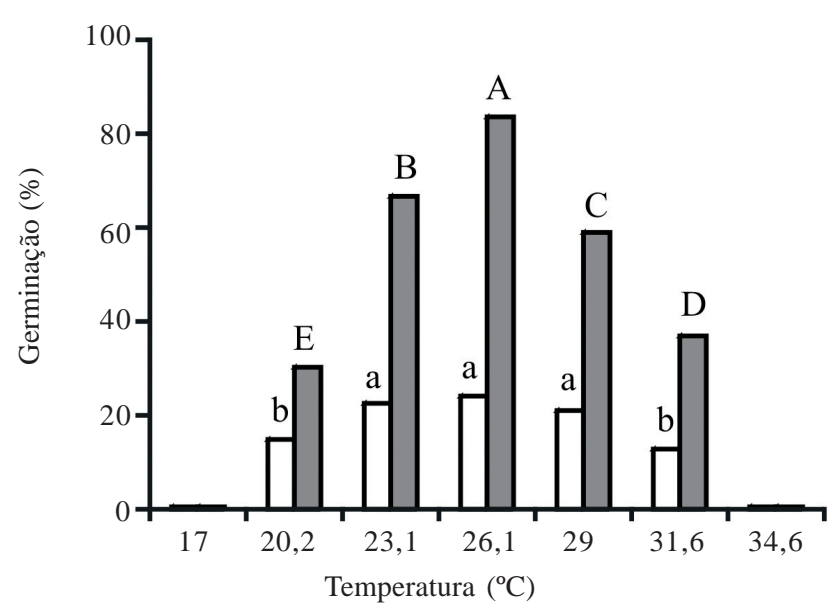

Figura 2. Dependência da temperatura da porcentagem $(\mathrm{G} \%)$ e velocidade média $\left(\mathrm{V}_{\mathrm{m}}\right)$ de germinação de sementes de Drymaria cordata (L.) Willd. ex Roem. \& Schult. (Cariophyllaceae) mantidas em bloco de gradiente térmico por 40 dias. Letras maiúsculas comparam as porcentagens e letras minúsculas comparam as velocidades médias de germinação (teste de Tukey, $\alpha=0,05 ; n=5$ ). Germinação nula a 17 e $34,6{ }^{\circ} \mathrm{C}(\square=\mathrm{Vm}$; $\square=\mathrm{G} \%)$.

Considerando-se os valores experimentais, a germinabilidade de Drymaria cordata apresentou temperatura ótima ao redor de $26^{\circ} \mathrm{C}$ (Fig. 2), diferente de espécies como Catharanthus roseus G. Don.(Cardoso 1999), cuja curva de dependência térmica da germinabilidade exibe um intervalo relativamente amplo de temperaturas ótimas. Quanto à velocidade média, não ocorreram diferenças significativas $(\mathrm{P}<0,05)$ entre 23 , 26 e $29^{\circ} \mathrm{C}$ (Fig. 2). Assim, considerando-se tanto a germinabilidade como a velocidade de germinação, $26^{\circ} \mathrm{C}$ pode ser definida como temperatura ótima para a germinação de sementes de $D$. cordata, estando as temperaturas de 20,2 e $23,1{ }^{\circ} \mathrm{C}$ na faixa infra-ótima, e 29 e $31,6^{\circ} \mathrm{C}$ na supra-ótima. A distribuição da germinação em diferentes temperaturas indica uma tendência de 
resposta linear tanto na faixa infra como na supra-ótima (Fig. 2), que poderia ficar mais bem caracterizada com o uso de um espectro de temperaturas mais amplo. Após 40 dias de experimento, a germinação foi nula às temperaturas de $13,9,17,0,34,6,36,8$; e $39,3{ }^{\circ} \mathrm{C}$, não tendo sido averiguada a presença de eventual dormência nas sementes não germinadas.

A distribuição das porcentagens de germinação acumuladas (Fig. 3) mostra que a mesma tende a se estabilizar em geral entre o $6^{\circ}$ e $7^{\circ}$ dias após o início do experimento, com exceção da temperatura de $31,6{ }^{\circ} \mathrm{C}$, cuja germinação atingiu o máximo após 12 dias. Considerando-se as germinabilidades relativamente baixas em temperaturas infra e supra-ótimas, para estimativa das velocidades de germinação $\left(1 / \mathrm{t}_{\mathrm{g}}\right)$, os tempos necessários para a germinação de cada fração percentual $\left(\mathrm{t}_{\mathrm{g}}\right)$ foram calculados apenas para as frações arbitrárias de 5, 10,20, 30 e 40\%. As velocidades de germinação correspondentes a cada fração $\left(1 / t_{5}\right.$ a $\left.1 / t_{40}\right)$ foram plotadas contra temperatura, podendo-se observar uma aparente resposta linear da velocidade de germinação das sementes de Drymaria cordata em função da temperatura (Fig. 4 e Tab. 1). Entretanto, deve-se salientar que, provavelmente pelo fato das regressões terem sido feitas
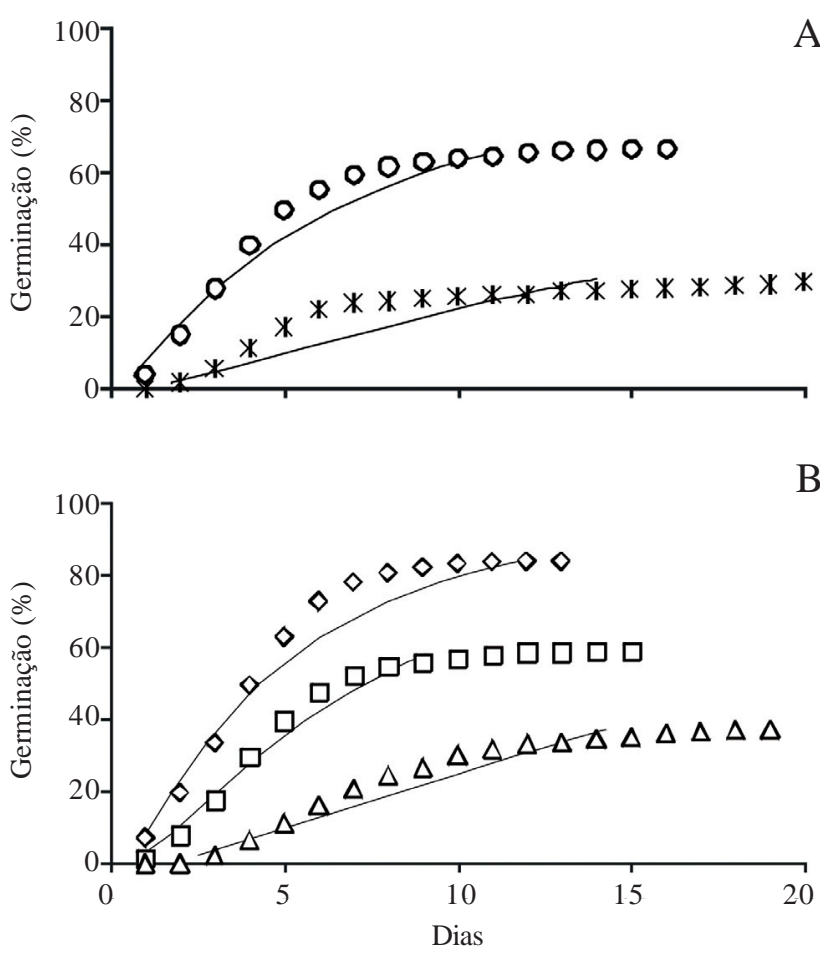

Figura 3. Curvas de germinação de sementes de Drymaria cordata (L.) Willd. ex Roem. \& Schult. (Cariophyllaceae) às temperaturas de 20,2 $(*), 23\left(\right.$ o), $26(\diamond), 29(\square)$ e $31,6{ }^{\circ} \mathrm{C}(\Delta)$. Símbolos indicam as porcentagens acumuladas médias observadas. A. Linhas contínuas representam as curvas descritas pelo modelo $t_{\mathrm{g}}=\left[10^{\text {(probit(g)-a). } \mathrm{\sigma}}\right] /\left(\mathrm{T}-\mathrm{T}_{\mathrm{b}}\right)$, com $\mathrm{a}=2,53 ; \sigma=0,56$ e $\mathrm{T}_{\mathrm{b}}=19,3$. B. Linhas contínuas representam as curvas descritas pelo modelo $\mathrm{t}_{\mathrm{g}}=\left[10^{\text {(probit(g)-a). } \sigma}\right] /\left(\mathrm{T}_{\mathrm{M}}-\mathrm{T}\right)$, com $\mathrm{a}=1,67 ; \sigma=0,45$ e $\mathrm{T}_{\mathrm{M}}=33,1$.

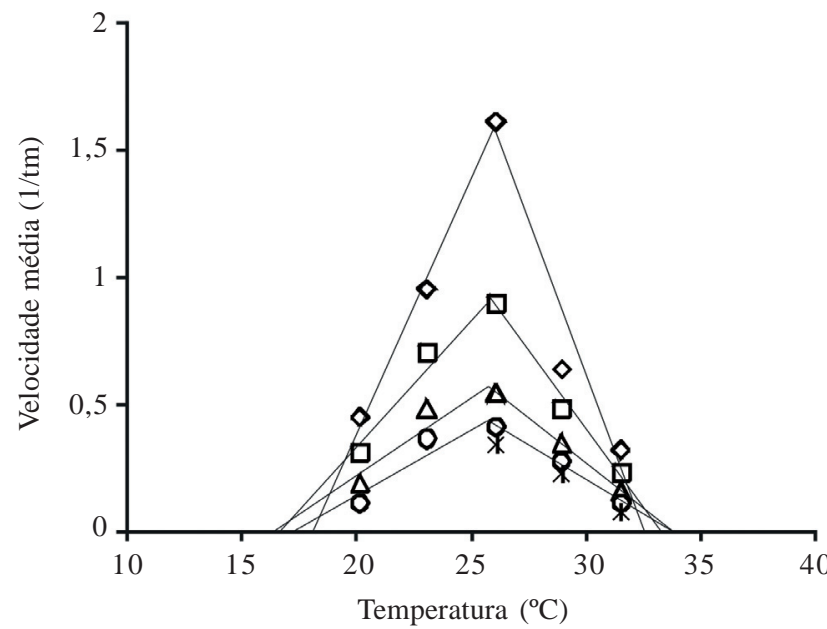

Figura 4. Relação entre temperatura e velocidades de germinação correspondentes às frações $5(\diamond), 10(\square), 20(\Delta), 30($ o) e $40 \%(*)$ de sementes de Drymaria cordata (L.) Willd. ex Roem. \& Schult. (Cariophyllaceae). Velocidade calculada como a recíproca de $t_{\mathrm{g}}$, isto é, do tempo (t) necessário para a germinação de uma fração percentual (g). Para o intervalo infra-ótimo não foi utilizada a fração $40 \%$. As respectivas equações são apresentadas na Tab. 1.

com apenas três temperaturas, alguns valores de $\mathrm{R}^{2}$ não foram significativos (Tab. 1). De qualquer modo, observa-se um aumento da velocidade com a temperatura na faixa infra-ótima e um decréscimo na supra-ótima (Fig. 4). Assumindo-se uma relação linear entre temperatura e velocidade de germinação, pode-se aplicar o modelo segundo o qual uma semente não germina a menos que receba uma determinada quantidade de grausdia acima de uma temperatura limítrofe mínima, ou $\mathrm{T}_{\mathrm{b}}$ (Larsen \& Bibby 2005). Acima da temperatura ótima, quando a germinação decresce com o aumento da temperatura (Fig. 2, 4), essa passaria a depender de quantidade de graus-dia abaixo de uma temperatura limítrofe máxima $\left(\mathrm{T}_{\mathrm{M}}\right)$ (Garcia-Huidobro et al. 1982) ou de variações da própria $T_{M}$ (Alvarado \& Bradford 2002). Quando $T_{M}$ varia com a fração percentual, ou seja, as retas do gráfico das velocidades contra temperaturas supra-ótimas são aproximadamente paralelas, deve-se usar o modelo probit $(\mathrm{g})=\mathrm{a}+\left[\left(\mathrm{T}+\theta / \mathrm{t}_{\mathrm{g}}\right) / \sigma_{\mathrm{TM}}\right]$ (Ellis et al. 1986) para tais temperaturas. Este último caso aparentemente não se aplica a $D$. cordata já que as curvas na faixa supra-ótima, a exemplo do que ocorreu na faixa infra-ótima, tendem a convergir na abscissa (Fig. 4). Valores de $\mathrm{T}_{\mathrm{b}}=19,3{ }^{\circ} \mathrm{C}$ e $\mathrm{T}_{\mathrm{M}}=33,5^{\circ} \mathrm{C}$ foram obtidos a partir do modelo descrito pela equação 5 , sugerindo que tais temperaturas representam, respectivamente, a mínima e máxima para a germinação de sementes de Drymaria cordata. O valor obtido para Tb é ligeiramente mais alto do que aqueles estimados a partir da extrapolação das retas de regressão das velocidades sobre as temperaturas supra-ótimas (Fig. 4, Tab. 1), 
Tabela 1. Parâmetros das equações lineares de ajuste das curvas de velocidade de germinação de diferentes frações percentuais de sementes de Drymaria cordata (L.) Willd. ex Roem. \& Schult. (Cariophyllaceae) em função da temperatura, na faixa infra-ótima e supra-ótima. Ensaio realizado em bloco de gradiente térmico. Valores nas colunas $T_{b}$ (temperatura base) e $T_{M}$ (temperatura máxima) representam as projeções das retas na abscissa $(\mathrm{y}=0)$. Na coluna de $\mathrm{R}^{2}$, asterisco indica valor significativo $\left(\mathrm{R}^{2}>0,976\right)$.

\begin{tabular}{|c|c|c|c|c|c|}
\hline Faixa & Fração & Equação & $\mathrm{R}^{2}$ & $\mathrm{~T}_{\mathrm{b}}$ & $\mathrm{T}_{\mathrm{M}}$ \\
\hline Infra-ótima & $\begin{array}{l}5 \% \\
10 \% \\
20 \% \\
30 \%\end{array}$ & $\begin{array}{l}\mathrm{y}=0,197 \mathrm{x}-3,568 \\
\mathrm{y}=0,099 \mathrm{x}-1,648 \\
\mathrm{y}=0,060 \mathrm{x}-0,987 \\
\mathrm{y}=0,057 \mathrm{x}-1,085\end{array}$ & $\begin{array}{l}0,995^{*} \\
0,959 \\
0,872 \\
0,854\end{array}$ & $\begin{array}{l}18,04 \\
16,65 \\
16,37 \\
18,97\end{array}$ & $\begin{array}{l}- \\
- \\
-\end{array}$ \\
\hline Supra-ótima & $\begin{array}{l}5 \% \\
10 \% \\
20 \% \\
30 \% \\
40 \%\end{array}$ & $\begin{array}{l}\mathrm{y}=-0,237 \mathrm{x}+7,719 \\
\mathrm{y}=-0,121 \mathrm{x}+4,050 \\
\mathrm{y}=-0,070 \mathrm{x}+2,393 \\
\mathrm{y}=-0,054 \mathrm{x}+1,829 \\
\mathrm{y}=-0,046 \mathrm{x}+1,562\end{array}$ & $\begin{array}{l}0,937 \\
0,988 * \\
0,999 * \\
0,993 * \\
0,992 *\end{array}$ & $\begin{array}{l}- \\
- \\
- \\
-\end{array}$ & $\begin{array}{l}32,51 \\
33,35 \\
33,89 \\
33,90 \\
33,54\end{array}$ \\
\hline
\end{tabular}

provavelmente devido ao fato de um número relativamente baixo de frações percentuais ter sido considerado. Marshall \& Squire (1996) observaram em sementes de Brassica napus L. que frações percentuais mais elevadas produzem valores mais elevados de $\mathrm{T}_{\mathrm{b}}$ em comparação com frações mais baixas, embora as diferenças não tenham sido significativas. Em $D$. cordata uma comparação deste tipo não pode ser realizada em virtude das análises terem se baseado apenas em frações de $40 \%$ ou menores.

Hardegree et al. (1999) observaram em gramíneas de clima temperado que $T_{b}$ pode variar principalmente em relação às frações percentuais maiores, ou seja, sementes que apresentam germinação mais lenta. Esse declínio de $\mathrm{T}_{\mathrm{b}}$ com o aumento da fração percentual não foi observado no presente trabalho, pois, como já mencionado, frações maiores não puderam ser consideradas. Temperatura base ao redor de $19^{\circ} \mathrm{C}$ pode ser considerada relativamente elevada se comparada, por exemplo, com espécies de clima temperado como Trifolium repens L., Lolium perenne L. e Cichorium intybus $\mathrm{L}$., cujas $\mathrm{T}_{\mathrm{b}}$ foram, respectivamente, $0{ }^{\circ} \mathrm{C}, 3.7^{\circ} \mathrm{C}$ e $1.2^{\circ} \mathrm{C}$ (Moot et al. 2000). Mesmo algumas espécies invasoras de culturas comuns no Estado de São Paulo, como Sida cordifolia L., S. glaziovii K.Schum. e S. rhombifolia L. apresentaram $\mathrm{T}_{\mathrm{b}}$ ao redor de $10^{\circ} \mathrm{C}$, portanto mais baixa do que a exibida por Drimaria cordata, e $\mathrm{T}_{\mathrm{M}}$ entre 35 e $40^{\circ} \mathrm{C}$ (Cardoso 1990; 1991; 1992). Para sementes de Hyptis suaveolens (L.) Poit., outra espécie invasora tropical, $\mathrm{T}_{\mathrm{b}}$ encontra-se ao redor de $15^{\circ} \mathrm{C}$ e $\mathrm{T}_{\mathrm{M}}$ entre 40 e $45^{\circ} \mathrm{C}$ (Felippe, comunicação pessoal). Assim, comparada com algumas outras espécies invasoras ou ruderais, $D$. cordata apresentou um intervalo ou "janela térmica" de germinação (diferença entre $T_{b}$ e $T_{M}$ ) relativamente estreito (aproximadamente $14{ }^{\circ} \mathrm{C}$ ).

Os parâmetros $\mathrm{T}_{\mathrm{b}} \mathrm{e} \mathrm{T}_{\mathrm{M}}$ foram usados na distribuição das porcentagens observadas de germinação na escala de graus-dia calculada de acordo com a equação 1 para temperaturas infra-ótimas (Fig. 5A), ou de acordo com a equação 2 para temperaturas supra-ótimas (Fig. 5B). Como mostrado no trabalho de Garcia-Huidobro et al. (1982) e em vários outros que se seguiram, esse procedimento faz com que as diferentes isotermas tendam a se sobrepor, indicando que a resposta das sementes à temperatura é função da distância entre a temperatura atual e as temperaturas cardeais extremas. Para efeito de comparação, é também apresentada a distribuição das porcentagens acumuladas de germinação em escala de graus-dia, cujos valores foram determinados de acordo com a equação 5. Observa-se uma boa correspondência entre os pontos e as respectivas curvas, particularmente para as porcentagens de germinação situadas no intervalo considerado para a elaboração do modelo, ou seja, até $30 \%$ para a faixa infra-ótima (Fig. 5A), e até $40 \%$ para a supra-ótima (Fig. $5 \mathrm{~B})$. De acordo com o modelo, na faixa infra-ótima obteve-se que, por exemplo, para $40 \%$ das sementes de Drymaria cordata germinarem são necessários aproximadamente 23 graus-dia. Substituindo-se esse valor na equação 1 obtêm-se $\theta_{40}=\left(T-T_{b}\right) t_{40}$. Considerando-se $\theta_{40}=23 \mathrm{e} \mathrm{Tb}=19$, a equação pode ser reescrita como $23=(\mathrm{T}-19) \mathrm{t}_{40}$. Nesse caso, tomando-se como exemplo a temperatura experimental de $20{ }^{\circ} \mathrm{C}$ seriam necessários cerca de 20 dias para que $40 \%$ das sementes de $D$. cordata germinassem; enquanto que para $\mathrm{T}=25{ }^{\circ} \mathrm{C}$ aproximadamente 4 dias seriam necessários para a germinação dos mesmos $40 \%$. Portanto, de acordo com o modelo, quanto mais afastada a temperatura experimental está de $\mathrm{T}_{\mathrm{b}}$, menor o tempo necessário para a germinação de uma dada fração percentual.

Também a partir do modelo descrito pela equação 5 , foram estimados os tempos $\left(\mathrm{t}_{\mathrm{g}}\right)$ esperados para a germinação em temperaturas infra-ótimas (Fig. 3A) e supra-ótimas (Fig. 3B), representados pelas linhas 

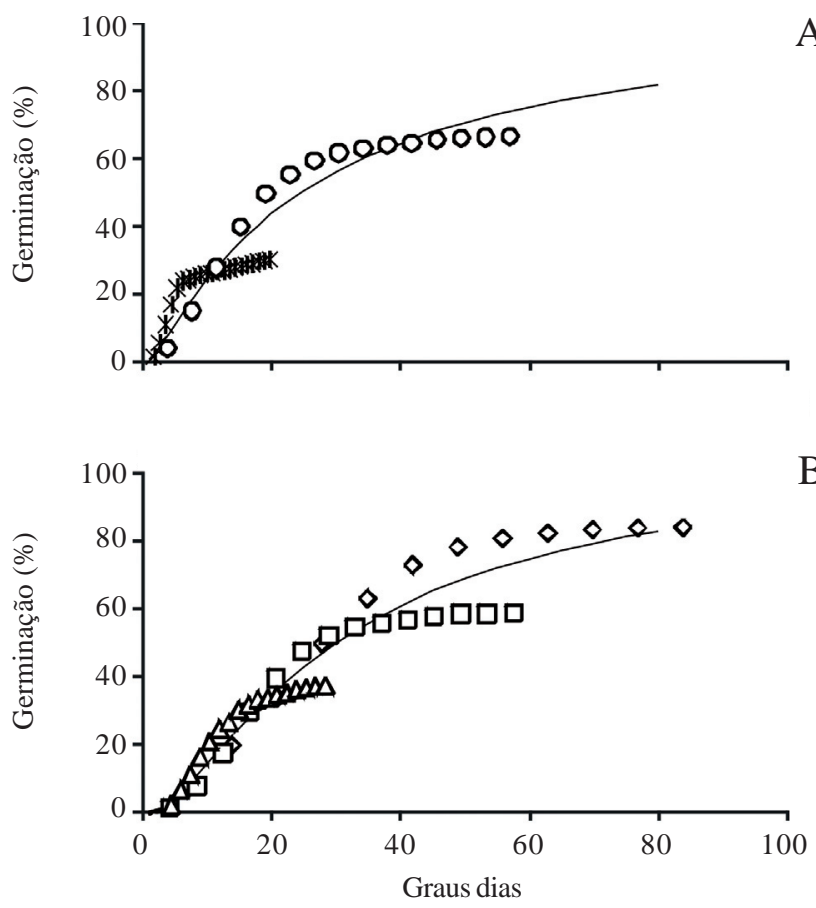

Figura 5. Germinação acumulada de sementes de Drymaria cordata (L.) Willd. ex Roem. \& Schult. (Cariophyllaceae) às temperaturas de $23\left(\right.$ o), $20(*), 26(\diamond), 29(\square)$ e $31,6{ }^{\circ} \mathrm{C}(\Delta)$, em escala de graus dia. A. Linhas contínuas representam as estimativas de graus dia segundo o modelo probit $(\mathrm{g})=\mathrm{a}+\left\{\log \left[\left(\mathrm{T}-\mathrm{T}_{\mathrm{b}}\right) \mathrm{t}_{\mathrm{g}}\right]\right\} / \sigma_{\theta}$, com $\mathrm{a}=2,53$, $\mathrm{Tb}=19,3$ e $\sigma=0,56$; símbolos representam os tempos reais de germinação multiplicados pelo fator $\left(\mathrm{T}-\mathrm{T}_{\mathrm{b}}\right)$. B Linhas representam o modelo probit $(\mathrm{g})=\mathrm{a}+\left\{\log \left[\left(\mathrm{T}_{\mathrm{M}^{-}} \mathrm{T}\right) \mathrm{t}_{\mathrm{g}}\right]\right\} / \sigma_{\theta}$, com $\mathrm{a}=1,69, \mathrm{~T}_{\mathrm{M}}=33,1$ e $\sigma=0,45$; símbolos representam os tempos reais de germinação multiplicados pelo fator $\left(\mathrm{T}_{\mathrm{M}}-\mathrm{T}\right)$.

contínuas. Observa-se que, com exceção da temperatura $\left(20,2{ }^{\circ} \mathrm{C}\right)$ mais próxima da temperatura base, o modelo descreveu com razoável precisão as curvas de germinação observadas. Com relação à temperatura ótima de $26^{\circ} \mathrm{C}$ (Fig. 3B), limítrofe entre as faixas sub e supraótima, não há diferença significativa $\left(\chi^{2}, \alpha=0.05\right)$ entre os pontos até aproximadamente $50 \%$ de germinação, sendo que a partir desse valor as porcentagens esperadas ficaram abaixo das observadas até a estabilização da curva. Segundo Hardegree et al. (1999), sub-populações de sementes que germinam mais rápido em temperaturas baixas provavelmente não são as mesmas que o fazem em temperaturas altas, situadas na faixa supra-ótima. Em Drymaria cordata, de qualquer modo, mais experimentos são necessários para se testar essa hipótese.

O efeito da temperatura sobre a germinabilidade de Drimaria cordata mostra que essa variável é bastante sensível a esse fator. De acordo com a literatura, temperaturas sub-ótimas podem tanto inibir (Moot et al. 2000), como não afetar a germinabilidade (Larsen \& Bibby 2005). Esses últimos autores reportam que, se a germinabilidade é inibida em temperaturas infra-ótimas, isso deve indicar que $T_{b}$ varia dentro da população, $a$ menos que o experimento tenha terminado antes da germinação se completar. Esse não deve ter sido o caso de $D$. cordata já que, no presente trabalho, as sementes ficaram nos germinadores por até 40 dias desde o início do experimento. Por outro lado, existe a possibilidade, que infelizmente não pode ser testada, das sementes mantidas em temperaturas infra-ótimas terem apresentado dormência secundária, que pode se instalar quando sementes não dormentes são mantidas por algum tempo em condições desfavoráveis à germinação (Labouriau 1983). De acordo com Hardegree et al. (1999), o pressuposto do modelo de graus-dia, segundo o qual a distribuição dos tempos de germinação é independente da temperatura, não é válido para sementes que exibem dormência em um ambiente com temperatura constante.

Com relação à faixa supra-ótima, apenas para efeito de comparação, testou-se também o modelo que considera $T_{M}$ variando com a fração percentual e "graus dia" $(\theta)$ constante, modelo esse que aparentemente se mostrou menos eficiente em descrever a germinação de Drymaria cordata nessa faixa (dados não apresentados). Entretanto, como uma análise comparativa mais acurada não foi feita, não é possível descartar a hipótese de que as diferenças entre os tempos de germinação isotérmica de $D$. cordata em temperaturas supra-ótimas possam ser causadas tanto pela variação de è, como pela variação de $\mathrm{T}_{\mathrm{M}}$.

Em suma, sementes de Drymaria cordata têm sua germinação estimulada pela luz branca (fotoblásticas) e germinam, em regime de temperaturas constantes, num intervalo térmico relativamente estreito. Assumindo-se que a velocidade de germinação de $D$. cordata apresenta uma variação linear em função da temperatura, e considerando que as temperaturas cardeais $\left(T_{b}\right.$ e $\left.T_{M}\right)$ estimadas para cada fração percentual variaram relativamente pouco, o modelo de graus-dia pode ser uma ferramenta válida para se estudar a dependência da temperatura da germinação dessa espécie, embora uma provável aquisição de dormência pelas sementes possa exigir a aplicação de outros modelos que não dependam das sementes apresentarem plena capacidade de germinação em diferentes regimes térmicos.

\section{Referências bibliográficas}

Alvarado, V. \& Bradford, K.J. 2002. A hydrothermal time model explains the cardinal temperatures for seed germination. Plant, Cell and Environment 25: 1061-1069.

Batlla, D. \& Benech-Arnold, R.L. 2006. The role of fluctuations in soil water content on the regulation of dormancy changes in buried seeds of Polygonum aviculare. Seed Science Research 16: 47-59.

Bradford, K. 2002. Applications of hydrothermal time to quantifying and modeling seed germination and dormancy. Weed Science 50: $248-260$. 
Bradford, K. 1995. Water relations in seed germination. Pp. 351-396. In: J. Kigel \& G. Galili (eds.). Seed development and germination. New York, Marcel Dekker Inc.

Brown, R.F. \& Mayer, D.G. 1988. Representing cumulative germination.2. The use of the Weibull function and other empirically derived curves. Annals of Botany 61: 127-138.

Cardoso, V.J.M. 1990. Germination studies on dispersal units of Sida rhombifolia L. Revista Brasileira de Botânica 13: 83-88.

Cardoso, V.J.M. 1991. Effects of temperature and seed coat on germination of Sida cordifolia. Ciência e Cultura 43: 306-308.

Cardoso, V.J.M. 1992. Temperature dependence on seed germination of a weed (Sida glaziovii - Malvaceae). Naturalia 17: 89-97.

Cardoso, V.J.M. 1999. Thermal-dependence of the germination of Catharanthus roseus seeds. Revista Brasileira de Fisiologia Vegetal 11: 41-49.

Dumur, D.; Pilbeam, C.J. \& Craigon, J. 1990. Use of the Weibull function to calculate cardinal temperatures in faba bean. Journal of Experimental Botany 41: 1423-1430.

Ellis, R.H.; Covell, S.; Roberts, E.H. \& Summerfield, R.J. 1986. The influence of temperature on seed germination rate in grain legumes. II. Intraspecific variation in chickpea (Cicer arietinum L.) at constant temperatures. Journal of Experimental Botany 17: 1503-1515.

Felippe, G.M. 1980. Germination of the light sensitive seeds of Cucumis anguria and Rumex obtusifolius: effects of temperature. New Phytologist 84: 439-448.

Garcia-Huidobro, J.; Monteith, J.L. \& Squire, G.R. 1982. Time, temperature and germination of pearl millet (Pennisetum typhoides) I. Constant temperature. Journal of Experimental Botany 33: 288-296.

Grundy, A.C.; Peters, N.C.B. \& Rasmussen, I.A. 2003. Emergence of Chenopodium album and Stellaria media of different origins under different climatic conditions. Weed Research 43: 163-176.

Gummerson, R.J. 1986. The effect of constant temperatures and osmotic potentials on the germination of sugar beet. Journal of Experimental Botany 37: 729-741.

Hardegree, S.P.; Van Vactor, S.S.; Pierson, F.B. \& Palmquist, D.E. 1999. Predicting variable-temperature response of non-dormant seeds from constant-temperature germination data. Journal of Range Management 52: 83-91.
Juroszek, P. \& Gerhards, R. Photocontrol of weeds. 2004. Journal of Agronomy and Crop Science 190: 402-415.

Labouriau, L.G. 1983. A germinação das sementes. Washington D.C, Secretaria Geral da Organização dos Estados Americanos, Programa Regional de Desenvolvimento Científico e Tecnológico. Série de Biologia. Monografia 24.

Labouriau, L.G. \& Agudo, M. 1987. On the physiology of seed germination in Salvia hispanica L. I. Temperature effects. Anais da Academia Brasileira de Ciencias 59: 37-56.

Larsen, S.U. \& Bibby, B.M. 2005. Differences in thermal time requirement for germination of three turfgrass species. Crop Science 45: 2030-2037.

Lorenzi, H. 1982. Plantas daninhas do Brasil. Nova Odessa, Edição do Autor.

Marshall, B. \& Squire, G.R. 1996. Non-linearity in rate-temperature relations of germination in oilseed rape. Journal of Experimental Botany 47: 1369-1375.

Miller, P.; Lanier, W. \& Brandt, S. 2001. Using Growing Degree Days to Predict Plant Stages. Montana State University Extension Service MT200103AG. Disponível em: http:// www.montana.edu/wwwpb/pubs/mt200103.pdf . (Acesso em: 4/abril/2008).

Moot, D.J.; Scott, W.R.; Roy, A.M. \& Nicholls, A.C. 2000. Base temperature and thermal time requirements for germination and emergence of temperate pasture species. New Zealand Journal of Agricultural Research 43: 15-25.

Qiu, J.; Bai, Y; Coulman, B. \& Romo, J.T. 2006. Using thermal time models to predict seedling emergence or orchardgrass (Dactylis glomerata L.) under alternating temperature regimes. Seed Science Research 16: 261-271.

Sokal, R.R. \& Rohlf, F.J. 1969. Biometry. São Francisco, W.H. Freeman and Co.

Tobe, K.; Zhang, L. \& Omasa, K. 2006. Seed germination and seedling emergence of three Artemisia species (Asteraceae) inhabiting desert sand dunes in China. Seed Science Research 16: 61-69.

Welbaum, G.E.; Bradford, K.; Yim, K.; Booth, D.T. \& Oluoch, M.O. 1998. Biophysical, physiological and biochemical processes regulating seed germination. Seed Science Research 8: 161-172. 